\title{
Lidil
}

Revue de linguistique et de didactique des langues

$64 \mid 2021$

Le passif dans la langue parlée

\section{Entre passif et anticausatif : étude des formes itCaCaC en arabe du Caire}

Between Passive and Anticausative: A Study of itCaCaC Forms in Cairo Arabic

Julie Haslé

\section{(2) OpenEdition}

1 Journals

Édition électronique

URL : https://journals.openedition.org/lidil/9613

DOI : $10.4000 /$ lidil. 9613

ISSN : 1960-6052

Éditeur

UGA Éditions/Université Grenoble Alpes

Édition imprimée

ISBN : 978-2-37747-315-1

ISSN : $1146-6480$

\section{Référence électronique}

Julie Haslé, "Entre passif et anticausatif : étude des formes itCaCaC en arabe du Caire », Lidil [En ligne], 64 | 2021, mis en ligne le 01 novembre 2021, consulté le 26 novembre 2021. URL : http:// journals.openedition.org/lidil/9613; DOI : https://doi.org/10.4000/lidil.9613

Ce document a été généré automatiquement le 26 novembre 2021.

(C) Lidil 


\section{Entre passif et anticausatif : étude des formes itCaCaC en arabe du Caire}

Between Passive and Anticausative: A Study of itCaCaC Forms in Cairo Arabic

Julie Haslé

\section{Introduction}

1 Le marquage du passif en arabe du Caire passe par un procédé de dérivation morphologique, la préfixation de it-. La dérivation des langues sémitiques en général, et pas seulement celle des verbes, repose sur une morphologie non concaténative, associée par Watson (2002) au modèle Root-and-Pattern, « racine et schème ».

2 De manière générale, la morphologie non concaténative utilise trois principaux procédés. La transfixation représente le modèle Root-and-Pattern dont le fonctionnement des langues sémitiques est l'archétype. Il s'agit de l'insertion d'une racine, une structure consonantique pour les langues sémitiques ${ }^{1}$, dans un morphème discontinu, le schème. L'alternance vocalique ou apophonie désigne la dérivation par modification des segments vocaliques qui composent le mot. Enfin, la réduplication produit le redoublement d'un segment ou d'un ensemble de segments. Ces trois procédés sont présents dans la dérivation des formes verbales en arabe.

3 Pour l'arabe du Caire, Woidich (2006) désigne cependant les formes verbales dérivées en découpant les préfixes qui sont présents sur des bases de dérivation. Ces bases de dérivation peuvent être la forme verbale simple (I), ou une des deux formes verbales qui en sont dérivées (II ou III). Ainsi, les formes verbales issues d'un second niveau de dérivation par préfixation sont décrites par leur modèle de formation. Trois formes verbales préfixées par it- sont associées à l'interprétation passive en arabe cairote :

\begin{tabular}{|l|l|l|}
\hline Base de dérivation & Formes préfixées \\
\hline - I ( $\mathrm{CaCaC})$ & $>$ & $\mathrm{t}-\mathrm{I}(\mathrm{itCaCaC})$ \\
\hline
\end{tabular}




\begin{tabular}{|l|l|l|}
\hline- II (CaCCiC ou CaCCaC) & $>$ & t-II (itCaCCiC ou itCaCCaC) \\
\hline- III (Ca:CiC) & $>$ & t-III (itCa:CiC) \\
\hline
\end{tabular}

4 Une des caractéristiques de ces formes verbales dérivées en arabe du Caire est de présenter une surdétermination, c'est-à-dire le fait pour une forme verbale de prendre en charge différentes interprétations. Concernant les formes t-I, t-II et $t$-III, il s'agit notamment du passif, de l'anticausatif, du réfléchi et du réciproque.

Cette surdétermination se joue à deux niveaux. On constate que plusieurs interprétations sont associées à une même forme, c'est-à-dire au modèle morphologique, le schème, qui est le procédé dérivationnel. Cependant, ces différentes interprétations ne se retrouvent pas sur tous les verbes qui sont formés sur ce modèle. Certains verbes admettent plusieurs interprétations, alors que d'autres n'en admettent qu'une.

6 Dans les exemples (1) et (2) suivants, le verbe it?afal reçoit deux interprétations différentes, « être fermé » et « se finir » :

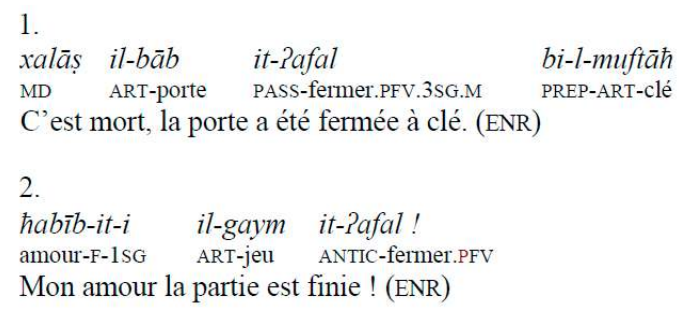

7 Ainsi, il semble qu'il faille différencier les valeurs associées au modèle de la manière dont les verbes dérivés les actualisent. L'un des aspects de la surdétermination des formes verbales dérivées en arabe du Caire est que les formes t-I reçoivent deux types d'interprétation, passive et anticausative.

8 Le passif désigne une réorganisation des actants par laquelle l'objet direct du verbe de la diathèse active est promu sujet du verbe de la construction à interprétation passive. Cette configuration entraine par rapport à la diathèse active la perte d'un actant, l'agent du procès, mais son existence est toujours sous-entendue (Rapatel, 2010; Hamma, 2020), et il peut être exprimé en fonction oblique par un groupe prépositionnel. En arabe cependant, il est traditionnellement admis que l'agent du procès n'est pas exprimable dans une construction à interprétation passive ${ }^{2}$.

9 L'interprétation anticausative présente le même type de construction que le passif puisqu'à nouveau, l'agent du procès n'est pas mentionné. Cependant, l'absence de l'agent est dans ce cas également sémantique, dans la mesure où le sujet est le lieu de réalisation du procès sans en être l'agent, et sans impliquer l'intervention d'un agent extérieur inconnu. Dès lors, le rôle sémantique d'expérienceur est attribué au sujet s'il est animé, afin de rendre compte de la manière dont il vit le procès, et de thème s'il est inanimé, insistant au contraire sur l'absence de sensations. Aussi, aucun agent n'est ni sémantiquement ni syntaxiquement récupérable par un groupe prépositionnel.

10 Ainsi, comme le souligne Kulikov (2011), les transformations décausative et passive produisent à priori le même type de constructions :

The anticausative has an important feature in common with the agentless passive: both entail the promotion of the initial Direct Object [patient] and the demotion of the initial 
Subject [agent], which accounts for their similar morphological marking in many languages.

(p. 392)

11 Du point de vue morphosyntaxique, les deux constructions auxquelles sont associées ces interprétations sont formellement identiques : un seul argument sujet d'un verbe préfixé par la marque verbale it- $(\mathbf{S} \mathbf{t}-\mathbf{I})^{3}$. Les deux constructions sont cependant différentes lorsque l'on prête attention aux rôles sémantiques qui sont associés à l'objet de la construction de la base de dérivation.

Du point de vue de leur interprétation, les verbes t-I se classent en trois catégories : ceux qui ne reçoivent qu'une interprétation passive, ceux qui ne reçoivent qu'une interprétation anticausative, et ceux qui peuvent recevoir les deux. Un premier mouvement de cette étude présentera la situation des verbes qui ne reçoivent qu'une interprétation passive, ainsi que les stratégies mises en place pour exprimer le passif lorsque la forme t-I n'est pas attestée.

Dans un second temps, nous nous intéresserons au cas dans lesquels la forme t-I est associée à l'interprétation anticausative, soit en étant la seule interprétation du verbe, soit en cohabitation avec l'interprétation passive. Nous présenterons les configurations dans lesquelles les deux interprétations sont attestées, afin d'identifier les caractéristiques qui orientent la lecture.

La distinction des deux interprétations se développe dans une troisième partie de l'étude par l'exploration des rapports qu'entretiennent les verbes t-I avec les notions d'agent et de cause. Nous observerons pour cela l'utilisation des formes t-I dans la construction analytique du causatif, qui met en avant leurs différences sémantiques, puis les constructions qui s'apparentent à des stratégies de récupération de l'agent.

\subsection{Sources des données}

Cette étude est basée sur des données issues d'enregistrements que nous avons réalisés et de notes que nous avons prises au Caire entre 2017 et 2018. À l'occasion de repas, de parties de jeux, de rencontres dans des cafés, ainsi que dans des réunions associatives, nous avons pu enregistrer des informateur.ice.s ayant entre 25 et 35 ans, ayant étudié à l'Université du Caire ou d'Ayn Shams dans des parcours universitaires en arabe. La mention (ENR) signale un exemple tiré de nos enregistrements, et la mention (NOTES) signifie qu'il est issu de notre prise de notes hors enregistrement.

Un deuxième type d'enregistrement compose nos données orales. Il s'agit d'enregistrements réalisés lors de séances de contes destinées à des enfants. Ces séances s'inscrivent dans un projet de refamiliarisation avec le patrimoine des contes égyptiens. Les exemples tirés des contes sont désignés comme (conte : titre du conte). Des données ont également été prélevées dans les chansons du groupe cairote Cairokee ${ }^{4}$. Les exemples tirés de ces corpus sont indiqués par le nom du groupe, le titre de la chanson dont l'exemple est issu et l'année de parution de l'album.

\section{Rapport des formes $\mathrm{t}-\mathrm{I}$ avec le passif}

18 Dans cette première partie de l'étude, nous observons le rapport des formes $t$-I avec l'interprétation passive. 


\subsection{Interprétation passive des formes $\mathrm{t}-\mathrm{I}$}

19 Un premier groupe de verbes t-I ne reçoivent que l'interprétation passive. Ainsi, le sujet de ces verbes conserve lors de la transformation syntaxique le rôle sémantique de [patient] qui est celui de l'objet du verbe I dans la diathèse active, ce qui engendre l'interprétation passive de la construction $\mathbf{S}$ [patient] $\mathbf{t}$-I.

Pour justifier l'existence de verbes qui ne reçoivent que l'interprétation passive, Kulikov (1998) avance l'idée de verbes pour lesquels le sens de la base de dérivation, c'est-à-dire la diathèse active $d u$ verbe, implique nécessairement une transitivité (inherently transitives) :

There is no need to argue that some transitive verbs can be both passivized and anticausativized (decausativized) (cf. (la-c), while some other verbs (which can be labeled inherently transitive) only allow for passivization. (p. 142)

Ainsi, le verbe itHaka « être raconté » est la forme préfixée par it- du verbe transitif Haka « raconter qqch ».

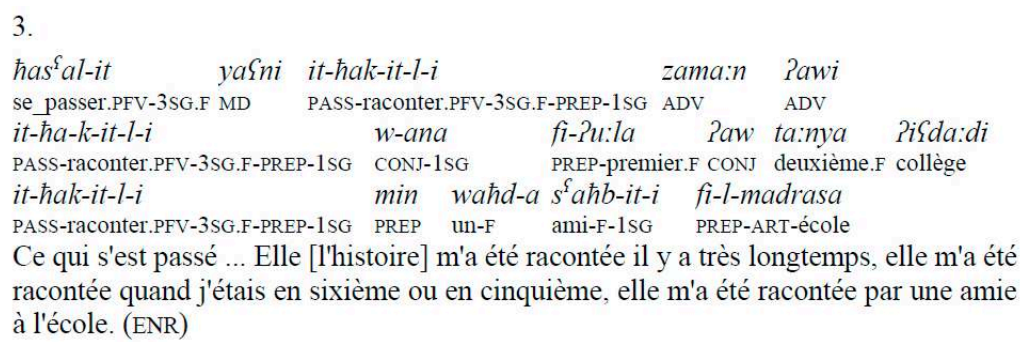

Ce verbe illustre l'idée d'une transitivité contenue dans l'activité décrite par le procès. En effet, lorsque le fait de raconter est rapporté, ce qui en est l'objet, l'histoire, participe nécessairement de la réalisation du procès.

\subsection{Expression du passif en l'absence de forme $t-I$}

Dans certains cas, cependant, la construction de la forme I ne permet pas la formation d'un verbe dérivé $t$-I. Le passif de la forme I ne peut pas être construit avec la forme $t$-I. Ces verbes n'ont pas de procédé morphologique pour construire la voix passive, ce qui amène à recourir à des procédés alternatifs.

\subsubsection{Forme I ditransitive}

Les verbes qui ont une base de dérivation I ditransitive ${ }^{5}$ n'ont pas de forme t-I attestée. Le passif de ces verbes est alors exprimé par une construction impersonnelle de la diathèse active, c'est-à-dire une construction qui correspond formellement à la diathèse active, mais pour laquelle le sujet n'a pas de référent.

4.

la? if-fant ${ }^{\S} a$ di idda-ha:-l-i ma ftar-et-ha:- -

ADV ART-Sac DEM.SG.F donner.PFV.3SG.M-3SG.F-PREP-1SG NEG acheter.PFV-1SG-3SG.F-NEG

Non ce sac-là m'a été donné, je ne l'ai pas acheté, pourquoi ? (ENR)

5

$\begin{array}{lllll}e: & d a & \text { if- } f a ? P a & \text { lissa ma } & \text { warr-u-ha:-f } \\ \text { INT } & \text { DEM.SG.M } & \text { ART-appartement ADV NEG } & \text { monter.PFV-3PL-3SG.F-NEG }\end{array} ?$

C'est quoi ça, l'appartement n'a toujours pas été montré ? (ENR) 

à laquelle s'adresse la question, le rôle d'objet ne peut être rempli que par un élément qui a justement pour référent cette question, comme suwa:l "question", ou Ha:ga "chose ", comme l'illustre l'exemple (6). Ainsi, la nature de cet objet est liée à la réalisation du procès, et ne peut pas être librement attribuée.

\subsubsection{Forme I intransitive} dérivation $\mathrm{I} \mathrm{CaCaC}$. Il existe également des verbes de forme $\mathrm{I}$, à vocalisation $\mathrm{i}-\mathrm{i}$ (CiCiC) intransitifs qui reçoivent une interprétation anticausative. Dans ce cas, aucune forme $\mathrm{t}$-I n'est attestée. L'interprétation anticausative bloque la dérivation d'une forme $\mathrm{t}$-I à interprétation passive. À travers le rapport de dérivation, la construction du passif se fait également entre des formes par l'intermédiaire d'autres verbes dérivés sur la même racine.

Nous constatons en effet que lorsque la forme I est intransitive, l'interprétation passive que l'on aurait observée avec une forme t-I est alors associée à la forme t-II dérivée sur la même racine. À partir de la forme I intransitive, une forme II ( $\mathrm{CaCCaC}$ ou $\mathrm{CaCCiC}$ ) est dérivée avec une interprétation agentive. La forme t-II en est alors le passif :

\begin{tabular}{|l|l|l|}
\hline I & hirib & « s'enfuir » \\
\hline II & harrab & « évader qqn » \\
\hline t-II & itharrab & « être évadé » \\
\hline
\end{tabular}


9.

fi? alf-a:n wi hdasfar na:s it-harrab-u min is-sign bi-dabbab-a:t PREP mille-D CONJ onze gens PASS-évader.PFV-3PL PREP ART-prison PREP-ART-tank-PL En 2011 des gens ont été évadés de prison par des tanks. (ENR)

\begin{tabular}{|l|l|l|}
\hline I & Sit ${ }^{\complement} i l$ & « tomber en panne » \\
\hline II & Sat $t^{\complement} t^{\complement} a l$ & « entraver, faire tomber en panne » \\
\hline $\mathrm{t}$-II & it $\left\{a t^{\complement} t^{\complement} a l\right.$ & « être entravé » \\
\hline
\end{tabular}

10.

law Pama:l-ik it-Sat $f^{\}}$al-it wi hla:m-ak farmil-it wi COND espoir-2SG.M PASS-entraver.PFV-3SG.F CONJ rêve.PL-2SG.M freiner.PFV-3SG.F CONJ guwwa:-k fa:yil $\hbar a-t a-$ Saddi PREP-2SG.M porter.PA.SG.M FUT-3SG.F-passer

Si tes espoirs sont entravés et que tes rêves sont freinés et que tu te sens triste, ça passera. (Cairokee, 2015 : kull ha:ga bitaGaddi)

\begin{tabular}{|l|l|l|}
\hline I & wisi & «s'élargir, s'agrandir » \\
\hline II & wassaS & « élargir » \\
\hline t-II & itwassaS & « être élargi » \\
\hline
\end{tabular}

11.

il-be:t it-wassas $\quad x a t^{\S} w a \quad b-x a t^{\S} w a$

ART-maison PASS-élargir.PFV.3SG.M pas PREP-pas

La maison a été agrandie petit à petit. (ENR)

La formation du passif par l'intermédiaire de la dérivation d'une autre forme permet d'illustrer la manière dont le système verbal de l'arabe du Caire s'organise. Cette organisation révèle des frontières poreuses entre les formes, au sein desquelles les verbes tirent leurs interprétations de l'existence des autres formes verbales sur la même racine. Dans le cas de l'absence d'une forme t-I, le verbe t-II reçoit une des interprétations possibles du modèle sur lequel il est formé, et cette interprétation est sélectionnée en conséquence de l'absence de la forme t-I.

\section{Du passif à l'anticausatif}

La forme verbale t-I est également associée à l'anticausatif.

\subsection{Interprétation anticausative des formes $\mathrm{t}-\mathrm{I}$}

Ainsi, un second groupe de verbes ne reçoivent que l'interprétation anticausative. L'objet de la base de dérivation I n'est alors pas associé au rôle sémantique [patient], 
mais à celui de [expérienceur]. Ce groupe concerne les verbes dont le procès relève de la sphère psychologique. Le référent du sujet est dès lors nécessairement animé.

Le verbe t-I itkasaf «se sentir embarrassé » est dérivé du verbe I kasaf « embarrasser quelqu'un ", pour lequel le sujet n'est pas vu comme un agent, mais comme le stimulus d'un processus interne. Dans l'exemple (12), notre informatrice précise que la présence imprévue d'une autre personne lors d'un rendez-vous a embarrassé le garçon qu'elle rencontrait.

12.

$$
\begin{aligned}
& \text { fihim bass it-kasaf } \\
& \text { comprendre.PFV.3SG.M CONJ ANTIC-embarrasser.PFV } \\
& \text { Il a compris mais il s'est senti embarrassé. (ENR) }
\end{aligned}
$$

Ainsi, dans ce cas comme dans celui des verbes qui ne reçoivent que l'interprétation passive, la conservation des rôles sémantiques des objets de la base de dérivation indique le lien avec la forme préfixée.

\subsection{Cohabitation des deux interprétations}

Ce lien entre construction de I et construction de t-I est moins visible dans le cas des verbes t-I qui entrent dans les deux constructions, à interprétation passive et à interprétation anticausative. À nouveau, les deux constructions sont formellement identiques.

Trois critères éclairent les modalités de cohabitation des deux interprétations: (1) le mode de réalisation du procès (physique/métaphorique); (2) les caractéristiques syntaxiques des verbes I et la spontanéité associée au procès.

\subsubsection{Mode de réalisation du procès}

Dans certains cas, la construction à interprétation anticausative est issue de la modification du mode de réalisation du procès par rapport à celui de la forme $t-I$ à interprétation passive. Nous parlons dans ce cas de réalisation métaphorique du procès, dans la mesure où $t-I$ à interprétation anticausative emprunte les traits sémantiques de la réalisation physique du procès. Cependant, l'action s'effectuant avec des actants ou dans des conditions qui ne permettent pas sa réalisation dans son acception première, celle-ci est alors réalisée par analogie avec la situation proposée par I.

Bybee (2013) note qu'une construction peut changer de sens dans l'usage, et dériver vers une nouvelle construction :

The conventionalization of implicature (or from the hearer's point of view, inference) is also well-known from grammaticalization research (Bybee, 1988b; Traugott, 1989; Bybee et al., 1994; Traugott and Dasher, 2002). It is thought that the frequent co-occurrence of an inference with a particular construction can lead to that inference being taken as part of the meaning of the construction. The originally inferential meaning can even replace the earlier meaning. (p. 5)

39 Ainsi, c'est par l'usage métaphorique d'un verbe que celui-ci vient à prendre progressivement un sens nouveau, qui dénote alors également un mode de réalisation différent du procès.

La situation de l'exemple (11) fait écho à celle de l'exemple (1). L'histoire qui y est racontée a resurgi après un temps d'oubli. 
13.
il-hika:ya di
id-dafan-it
id-dafan-it
$x a: l i s^{\varsigma}$
ART-histoire DEM.SG.F ANTIC-enterrer.PFV-3SG.F ANTIC-enterrer.PFV-3SG.F ADV
Cette histoire s'est enfouie, elle s'est complètement enfouie. (ENR)

Dans ce cas, il semble que le mode de réalisation par métaphore se soit imposé à titre égal de la réalisation première du procès, au point que la base de dérivation soit elle aussi réinterprétée avec une alternative métaphorique.
3 La base de dérivation xana? est alors elle aussi réinterprétée sur ce mode de réalisation : « donner à qqn le sentiment d'étouffer ».

15.

$$
\begin{array}{llll}
\text { mawd } d^{\S} u: \mathcal{G} & \text { ig-gawa:z } & \text { bi-yi-xnu?-ni } & \text { giddan } \\
\text { sujet } & \text { ART-mariage } & \text { IND-3-étouffer.IPFV-1SG } & \text { ADV } \\
\text { Le sujet du mariage ça m'oppresse vraiment. } &
\end{array}
$$

D'autres verbes proposent également les deux types de modes de réalisation de la base de dérivation :

\begin{tabular}{|l|l|l|l|}
\hline & I & & t-I \\
\hline réalisation physique & Pafal : «fermer qqch » & $>$ & it?afal : « être fermé » \\
\hline $\begin{array}{l}\text { réalisation } \\
\text { métaphorique }\end{array}$ & Pafal : « déprimer qqn» & $>$ & it?afal : « déprimer » \\
\hline réalisation physique & Hafar fi: « insérer qqch dans qqch» & $>$ & $\begin{array}{l}\text { itHafar fi : «être inséré dans } \\
\text { qqch }\end{array}$ \\
\hline $\begin{array}{l}\text { réalisation } \\
\text { métaphorique }\end{array}$ & $\begin{array}{l}\text { Hafar fi: «immiscer qqch dans } \\
\text { qqch }\end{array}$ & $>$ & $\begin{array}{l}\text { itHafar fi: «s'immiscer dans } \\
\text { qqch » }\end{array}$ \\
\hline
\end{tabular}



soit une interprétation passive, soit une interprétation anticausative. Dans ces deux cas, les rôles sémantiques de la construction de I sont conservés, puisqu'il y a deux versions de I, qui se différencient par leurs modes de réalisation.

Ainsi, il y a des cas dans lesquels la lecture anticausative de t-I a une forme I, et d'autres dans lesquelles elle n'en a pas.

\subsubsection{Labilité verbale}

Le passage de l'interprétation passive à l'interprétation anticausative a également lieu dans un second cas de figure. Certaines bases de dérivation I appartiennent à la catégorie des verbes labiles, qui peuvent être construits de manière transitive ou intransitive. Creissels (2006) décrit le comportement syntaxique de ces verbes labiles:

On désigne parfois comme labiles les verbes transitifs dont l'emploi intransitif implique que le rôle sémantique du sujet soit modifié de la même façon qu'auprès d'une forme verbale passive, ou auprès d'une forme moyenne de sens décausatif. Avec les verbes transitifs prototypiques, dont le sujet représente un agent, ce type de comportement signifie qu'en l'absence d'un objet, le sujet reçoit le rôle de patient ou de siège du procès. Le français a ainsi un nombre relativement élevé de verbes comme baisser, dont l'emploi dans Le prix des tickets de bus a baissé peut être qualifié de décausatif par rapport à l'emploi transitif illustré par La compagnie de transport a baissé le prix des tickets de bus. (p. 4)

L'objet du verbe non préfixé peut aussi en être le sujet, sans modifier le mode de réalisation du procès. Woidich (2006) note déjà cette caractéristique de certains verbes I transitifs en arabe du Caire :

Dans le cas d'un certain nombre de verbes transitifs des formes I et II, le complément d'objet peut être omis, ce qui entraine un sens médio-passif. Le sujet n'est plus un agent mais un expérienceur, i.e. quelque chose lui arrive. Un $b \bar{a} S$, yibī h h 'vendre quelque chose' signifie alors $b \bar{a} S$, yibī 'se vendre', par exemple : il Pusțuwāna bāYit kitīr 'le disque s'est bien vendu' [SB]. Et tant d'autres verbes, y compris ceux de la forme $\mathrm{II}^{6}$. (p. 255)

On constate que lorsqu'un verbe I a cette caractéristique, alors la forme t-I peut avoir une lecture passive et également une lecture anticausative sans modification du mode de réalisation du procès. Dès lors, il existe un rapport de concurrence entre I et t-I, puisque les deux formes semblent synonymes dans leurs emplois anticausatifs. Nous proposons d'illustrer ce cas avec les exemples du verbe I Pafal «fermer» et de la forme t-I it? afal qui en est dérivée.

I transitif : ?afal : « fermer qqch »

16.

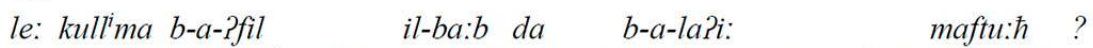
INT CONJ IND-1SG-fermer.IPFV ART-porteDEM.SG.MIND-1SG-trouver.IPFV.SG.M ouvert.PP.SG.M Pourquoi est-ce qu'à chaque fois que je ferme cette porte je la retrouve ouverte ? (ENR)

I intransitif : ?afal : «fermer»

17.

il-mahall-a:t bi-yi-?fil-u is-sa:Sa Safara hina

ART-magasins-PL IND-3-fermer.IPFV-3PL ART-heure dix ADV

Les magasins ferment à dix heures ici. (ENR)

t-I passif : it?afal : «être fermé » 
18.

xala:s $s^{5}$-ba:b it-Pafal bi-l-mufta: $\hbar$

C'est mort, la porte a été fermée à clé. (ENR)

t-I anticausatif : it? afal : « se fermer »

19.

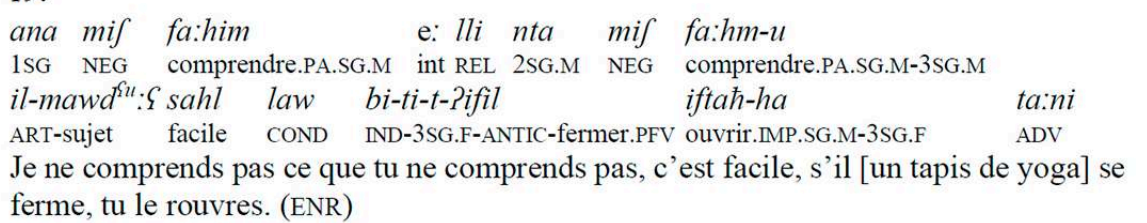

20.

il-mahall illi ftare:-na minn-u l-lamba it-Pafal

ART-magasin REL acheter.PFV-1PL PREP-3SG.M ART-lampe ANTIC-fermer.PFV.3SG.M

Le magasin dans lequel nous avons acheté la lampe a fermé (définitivement). (ENR)

21.

22.

Cependant, la concurrence entre les formes I et $\mathrm{t}$-I n'est dans certains cas qu'apparente. Nous relevons en effet par exemple une différence d'usage entre les formes I et $\mathrm{t}$-I des exemples (18) et (20) Si les deux décrivent la fermeture du magasin, avec le verbe I intransitif il s'agit de la fermeture quotidienne, alors que la forme t-I annonce la fermeture définitive.

\subsubsection{Spontanéité du déclenchement de l'action}

Il existe enfin un dernier cas de figure dans lequel une forme t-I peut recevoir les deux interprétations. Le mode de réalisation n'est pas en cause, puisqu'il s'agit bien dans ces acceptions de la réalisation physique du phénomène.

La spontanéité du déclenchement de l'action désigne l'absence d'une cause externe, sans impliquer la volonté du sujet dans la réalisation du procès, qui en est cependant le lieu de réalisation. Cette spontanéité est illustrée par l'exemple (22), qui s'oppose alors à l'exemple (21) dans lequel une cause externe est sous-entendue.

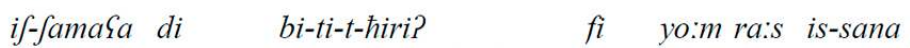
ART-bougie DEM.SG.F IND-2SG.F-PASS-bruler.IPFV PREP jour tête ART-année On brule cette bougie le jour du nouvel an. (NOTES) xud-i l-kabi:r mif ha-yi-t-hiris Sala t $t^{\varsigma} u: l$ prendre.IMP-F ART-grand NEG FUT-3SG.M-ANTIC -bruler.IPFV LOC Prends le grand [morceau de bois], il ne brulera pas tout de suite. (ENR)

À notre connaissance, les verbes I à partir desquels sont dérivés ces verbes $t$-I à lecture anticausative n'ont pas la caractéristique de labilité observée pour certains verbes I dans la section précédente. Il est cependant notable que ces verbes décrivent des procès auxquels peut être associée une notion de spontanéité du déclenchement de l'action, en l'absence de cause externe.

\section{Rapport à la cause}

La différence fondamentale entre les deux interprétations passive et anticausative est leur rapport à la cause. Dans le cas du passif, bien que la cause ne soit pas exprimée, son 
existence est sous-entendue. Au contraire, l'anticausatif s'applique à des procès internes auxquels aucune cause externe n'est associée. Ainsi, cette différence se retrouve dans les constructions dans lesquelles elles sont utilisées, ce qui permet à nouveau de les distinguer dans l'usage.

Cette dernière partie propose deux manières de regarder l'implication d'une cause dans la réalisation du procès, à travers le rôle du sujet de la construction analytique du causatif, et en observant la nature des compléments introduits par les prépositions min et $b i$.

\subsection{Construction analytique du causatif}

61 La construction analytique du causatif permet d'identifier et de distinguer les deux types d'interprétations reçues par les verbes $t$-I. Le causatif désigne une construction dans laquelle le sujet cause par l'intermédiaire d'un autre actant, souvent associé à l'objet de la construction, la réalisation d'un procès.

DeLancey (1984) présente deux types de constructions causatives: une construction causative par intermédiaire (mediated causation), et une construction causative directe (direct causation). La construction causative directe est marquée morphologiquement sur la forme verbale ou sur les désignations casuelles des actants. La construction causative par intermédiaire est quant à elle prise en charge par un élément syntaxique :

Typically, mediated causation is coded by a periphrastic construction with a distinct causative verb, while direct causation is coded by a morphologized or even lexicalized distinction. (p. 182)

L'arabe du Caire présente les deux types de constructions. D'une part, le modèle morphologique II ( $\mathrm{CaCCaC} / \mathrm{CaCCiC}$ ) a une valeur causative qui en fait le procédé de causation directe en arabe du Caire et en arabe de manière générale. Parmi les valeurs de forme II, Woidich (2006) relève celle de « causatif (faire, amener, causer) » (p. 72).

En parallèle, il est possible d'avoir recours à la construction analytique du causatif, qui correspond à la construction causative par intermédiaire (mediated causation). Celle-ci utilise le verbe xalla, qui a pour sens premier « laisser».

23.

$$
\begin{aligned}
& \text { ana kida mif h-a-xalli:-hum yi-Saks-u:-ni ma yi-nfaS-f } \\
& \text { 1SG DEM NEG FUT-1SG-laisser.IPFV-3PL 3-draguer-PL-1SG NEG 3-être possible.IPFV-NEG } \\
& \text { De toute façon je ne vais pas les laisser me draguer comme ça, impossible. (ENR) }
\end{aligned}
$$

Lorsqu'elle est utilisée avec un verbe t-I qui ne peut être utilisé qu'avec une construction à interprétation passive, elle modifie la manière dont l'implication de l'agent du verbe I dans le procès est perçue. En effet, contrairement au sujet du verbe I, celui du verbe xalla est perçu comme la cause externe mais peut ne pas avoir eu de rapport direct avec la réalisation du procès. La construction peut alors se traduire 'il a été fait en sorte que...'.

Dans l'exemple (24), le verbe iddafaS "être payé», utilisé avec la construction analytique du causatif, ne permet pas d'affirmer si le sujet de xalla a lui-même ou non remboursé la dette dont il est question, ou s'il a chargé quelqu'un de le faire. 


$$
24 .
$$

67 Ainsi, cette phrase n'a pas le même sens que l'utilisation du verbe de forme I, dans laquelle le sujet est effectivement celui qui réalise le procès.

$\mathrm{Au}$ contraire, lorsqu'un verbe t-I reçoit une interprétation anticausative, alors la construction analytique du causatif est en concurrence avec l'utilisation de la base de dérivation de forme I, ici le verbe raSab « effrayer qqn » :

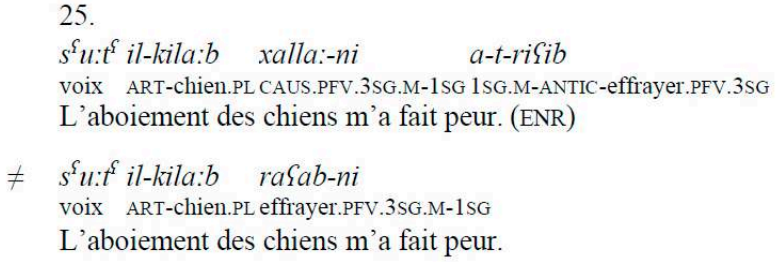

69 Les deux phrases sont interprétées de la même façon, car les sujets du verbe de forme I et celui de l'auxiliaire du causatif xalla sont considérés comme ayant le même rôle de cause externe.

70 Ainsi, une différence observable lorsqu'une forme t-I à lecture anticausative est utilisée avec la construction analytique du causatif ne porte pas sur l'implication du sujet dans la réalisation du procès, mais sur l'intentionnalité qui lui est conférée. La nature du sujet de l'auxiliaire du causatif peut créer une ambigüité sur l'intentionnalité à réaliser le procès.

71 Quand on suppose une volonté du sujet, la construction analytique peut expliciter ou intensifier l'intentionnalité de l'agent à réaliser le procès :

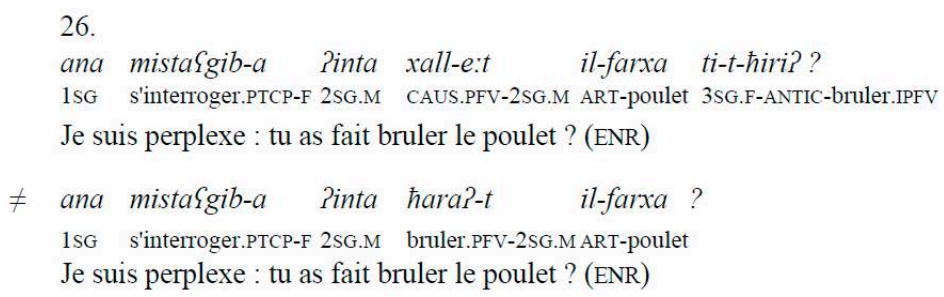

Le contexte de l'enregistrement nous indique qu'il s'agit ici d'une accusation visant l'intention du sujet. Mais d'après nos informateur.ice.s, dans d'autres contextes, elle pourrait signifier que ce n'était pas l'intention du sujet, mais que celui-ci n'a pas fait ce qu'il fallait pour que le poulet ne brule pas.

73 Cette ambigüité dépend notamment de la possibilité d'attribuer une volonté au sujet. Dans le cas de l'exemple (26), l'interprétation volontaire est possible parce que le sujet est humain. Dans le cas d'un sujet non humain, comme c'est le cas dans l'exemple (25), le doute ne subsiste cependant pas. 
27.

il-hawa: huwwa illi xalla if-fibba:k yi-t-Pifil

ART-vent 3SG.M REL CAUS.PFV.3SG.M ART-fenêtre 3-PREF-fermer.IPFV

C'est le vent qui a fermé la fenêtre. (ENR)

\# il-hawa huwwa illi Pafal if-fibba:k

ART-vent 3SG.M REL fermer.PFV.3SG.M ART-fenêtre

C'est le vent qui a fermé la fenêtre. arabe littéral moderne :

Enfin, l'agent étant par définition non spécifié, toute expansion agentive équivalente à celle du "complément d'agent» de la grammaire française est, en principe, exclue, même si l'arabe littéral moderne tend à faire usage de groupes plus ou moins calqués sur les syntagmes agentifs français ou anglais. (p. 13)

Le vent est identifié comme la cause, mais aucune volonté de réaliser l'action ne peut lui être associée.

Ainsi, l'observation de l'utilisation des formes t-I dans la construction analytique du causatif permet de soulever deux manières de distinguer les interprétations passive et anticausative. En d'autres termes, les deux interprétations de t-I nous permettent de voir les sujets de xalla de deux manières différentes.

Sur le plan sémantique, on constate une différence de traitement des deux interprétations du point de vue de l'implication directe du sujet dans la réalisation de l'action. Si celle-ci est remise en cause dans le cas de l'utilisation des formes t-I à interprétation passive, elle ne l'est pas lorsque la forme t-I reçoit une lecture anticausative. Seule une différence dans l'intentionnalité du procès peut être décelée. Ainsi, la construction analytique du causatif n'est pas en concurrence avec la forme I de la même manière en fonction de la lecture de la forme t-I qui y est utilisée. Si le verbe $t-I$ a une interprétation passive, on n'observe pas de concurrence, alors que s'il reçoit une interprétation anticausative, il existe une concurrence avec la base de dérivation.

Cette différence de valeur associée à la construction analytique du causatif en fonction de l'interprétation de la forme t-I s'explique par le rapport qu'entretiennent passif et anticausatif aux notions d'agent et de cause. Dans le cas des verbes qui reçoivent une lecture anticausative, le sujet du verbe I et celui de l'auxiliaire du causatif ont le même statut vis-à-vis du procès, à savoir celui de cause externe. Dans les deux cas, ils ne sont pas agent du procès, déclencheurs d'un procès interne. En revanche, dans le cas des verbes qui reçoivent une lecture passive, la cause externe de la construction causative ne correspond pas forcément à l'agent de la diathèse active, puisque contrairement à l'anticausatif, le passif a un agent, une cause externe non exprimée mais sousentendue.

\subsection{Récupération de l'agent}

On considère traditionnellement qu'il n'existe pas de stratégie de récupération de l'agent en arabe. Cependant, Brahim (1996) nuance ce postulat au vu des usages en ous avons observé la situation en arabe du Caire. Grâce à la comparaison des constructions à interprétations passive et anticausative, il est possible de rejeter l'idée d'un complément d'agent. En effet, deux constructions font penser à un moyen de 
récupérer l'agent avec un verbe à interprétation passive. Il s'agit de syntagmes nominaux introduits par les prépositions min et bi.

De manière générale, la préposition min indique notamment la source du procès ; dans le cas de l'exemple (28), il s'agit du matériau utilisé dans la confection du vêtement.

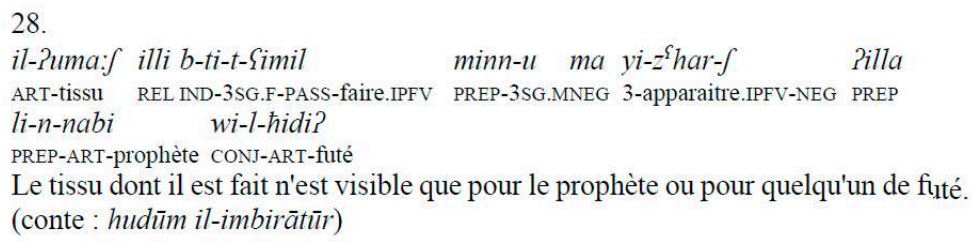

81 Le syntagme prépositionnel avec min introduit également le stimulus des verbes psychologiques.

Ainsi, dans l'exemple (29), le pronom - $u$ introduit par la préposition min renvoie à la personne à partir de laquelle le dégout a été déclenché.

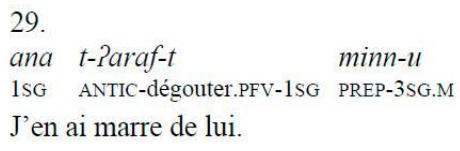

Cette utilisation de la préposition min est ambigüe, car il pourrait s'agir d'un procédé de récupération de l'agent, qui correspondrait au sujet de la forme I. Cependant, des verbes I intransitifs à lecture anticausative sont utilisés dans la même construction. Le verbe zihi? fait partie des verbes I de forme $\mathrm{CiCiC}$ intransitifs qui reçoivent une lecture anticausative. On le trouve construit avec le syntagme prépositionnel introduit par min.

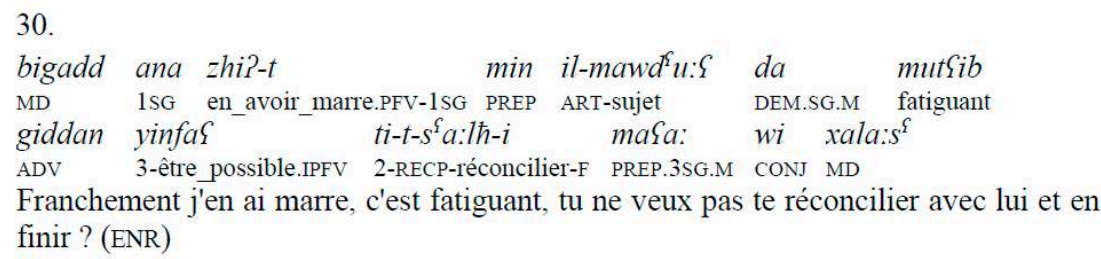

On en déduit que lorsque le verbe t-I it?araf est utilisé avec un groupe prépositionnel introduit par min comme dans l'exemple (29), alors de la même manière son objet est considéré comme la source d'un procès interne au sujet du verbe, qui s'accomplit donc sans cause externe.

La préposition bi met également le procès en relation avec un élément extérieur, mais celle-ci est cette fois comprise comme l'instrument de sa réalisation. L'exemple (31) illustre la valeur instrumentale de la préposition $b i$, utilisée avec le participe passif marbu: $t^{\complement}$, introduisant l'instrument, l'élastique, par lequel sont attachés les cheveux.

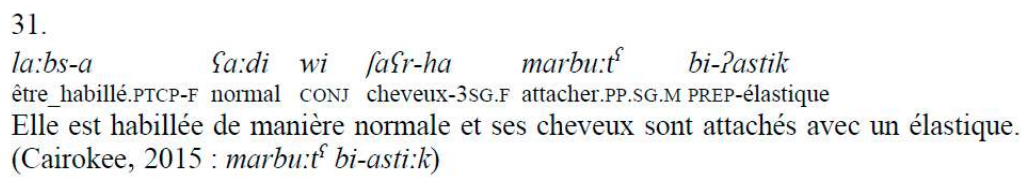

Ainsi, les verbes préfixés suivants utilisent cette même valeur instrumentale pour exprimer un élément qui participe au procès. Celui-ci intervient avec des verbes à lecture passive comme le verbe itfagal : «être occupé ».

32.

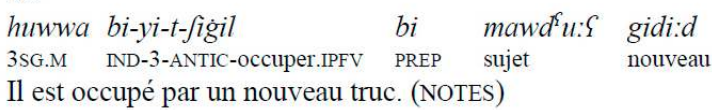


87 À nouveau, c'est la comparaison avec la construction à lecture anticausative qui permet de déduire que l'élément introduit par la préposition bi n'est toujours pas identifié comme la cause. En effet, ce syntagme intervient également avec des verbes à lecture anticausative, comme c'est le cas du verbe ithabal "devenir fou ", qui peut être construit avec un syntagme prépositionnel introduit par bi: ithabal bi «devenir fou à cause de qqn ou qqch ».

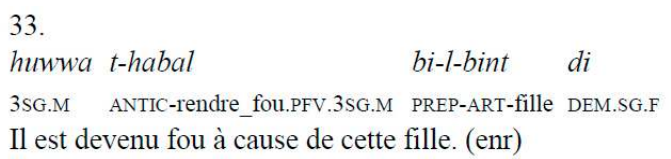

Tout comme avec la préposition min, l'utilisation du syntagme introduit par bi avec un verbe recevant une lecture anticausative empêche de considérer l'objet introduit par ce syntagme comme l'agent du procès.

89 Ainsi, par comparaison des usages qui sont faits de ces deux syntagmes prépositionnels avec des verbes recevant les lectures passive et anticausative, on constate qu'il ne s'agit pas d'un procédé de récupération de la cause, dans la mesure où il est impossible de récupérer une cause pour un procès à valeur anticausative.

\section{Conclusion}

Deux lectures possibles des formes t-I intransitives ont été identifiées. Une lecture passive, issue de la transformation passive de la construction de I, conserve le rôle sémantique patient de l'objet de la construction active correspondante; et une lecture anticausative, issue de la transformation décausative de la construction de I, modifie en expérienceur le rôle sémantique patient de l'objet de la construction active correspondante.

91 Ainsi, malgré leurs similarités syntaxiques, deux points principaux séparent ces deux lectures : l'existence d'une cause externe, et la modification du rôle du sujet. Il a été vu que certaines formes t-I qui reçoivent une lecture anticausative sont dérivées d'une base I qui ne présente pas le même mode de réalisation. La transformation syntaxique modifie le mode de réalisation.

92 Ces deux lectures des formes t-I permettent donc d'illustrer les deux types de transformations syntaxiques prises en charge par le préfixe $t$ - lorsqu'il est utilisé avec une base de dérivation $\mathrm{I}$.

93 Un dernier point porte sur les conditions de dérivation des formes $t$-I. Nous avons en effet constaté qu'aucune forme t-I n'existe en l'absence de la base de dérivation I correspondante. Dès lors, il semble qu'un rapport de dérivation fonctionnel existe bien entre les deux formes. Notre hypothèse est que le lien particulier entre base de dérivation I et forme t-I tient à la transformation passive marquée par le procédé de dérivation par préfixation. En effet, le procédé de dérivation est alors l'aspect morphologique de l'interface morphosyntaxique de la lecture passive. Elle décrit alors un rapport syntaxique actif lorsque le préfixe it- est utilisé sur une base de dérivation I. 


\section{BIBLIOGRAPHIE}

BRAHIM, Ahmed. (1996). L'occultif, hypothèse pour un traitement trans-linguistique du « passif » et des structures apparentées. Association tunisienne de linguistique.

BYBEE, Joan Lea. (2013). Usage-Based Theory and Exemplar Representations of Constructions. Dans T. Hoffmann \& G. Trousdale (dir.), The Oxford Handbook of Construction Grammar (p. 49-69). Oxford University Press.

CREISSELS, Denis. (2006). Syntaxe générale : une introduction typologique. Tome 1 : Catégories et constructions. Hermès sciences / Lavoisier.

DeLANCEY, Scott. (1984). Notes on Agentivity and Causation. Studies in Language. International Journal sponsored by the Foundation "Foundations of Language", 8(2), 181-213.

HAMMA, Badreddine. (2020). Pourquoi ne peut-on pas se passer de l'agent passif dans une vraie conversation ? Dans C. Jamil, O. Lassâad \& H. Layla (dir.), Le dialogue et la conversation à la croisée des approches (p. 87-103). Latrach Édition.

Kulikov, Leonid. (1998). Passive, Anticausative and Classification of Verbs: The Case of Vedic. Dans L. Kulikov \& H. Vater (dir.), Typology of Verbal Categories: Papers Presented to Vladimir Nedjalkov on the Occasion of his 70th Birthday (p. 139-154). Max Niemeyer Verlag.

Kulikov, Leonid. (2011). Voice Typology. Dans S. Jae Jung (dir.), The Oxford Handbook of Linguistic Typology (p. 368-398). Oxford University Press.

RAPATEL, Philippe. (2010). À la recherche de l'agent masqué : l'agentivité implicite. Cahiers du Laboratoire de recherche sur le langage, Construction de la cohérence, construction du sens, 4, 1-9. WATson, Janet. (2002). The Phonology and Morphology of Arabic. Oxford University Press.

WoIDICH, Manfred. (2006). Das Kairenisch-Arabische. Eine Grammatik. Otto Harrassowitz Verlag.

\section{ANNEXES}

\section{Abréviations de la glose}

\begin{tabular}{|l|l|l|l|l|l|}
\hline 1 & première personne & DEM & démonstratif & NEG & négation \\
\hline 2 & deuxième personne & FEM & féminin & PA & participe actif (forme I) \\
\hline 3 & troisième personne & FUT & futur & PASS & passif \\
\hline ADV & adverbe & IMP & impératif & PFV & perfectif \\
\hline ANTIC & anticausatif & IND & indicatif & PL & pluriel \\
\hline ART & article & INDF & indéfini & PP & participe passif (forme I) \\
\hline CAUS & causatif & INT & interrogatif & PREP & préposition \\
\hline
\end{tabular}




\begin{tabular}{|l|l|l|l|l|l|}
\hline COND & conditionnel & IPFV & imperfectif & PTCP & participe \\
\hline CONJ & conjonction & LOC & locution & REL & relatif \\
\hline COP & copule & M & masculin & SG & singulier \\
\hline D & duel & MD & marqueur discursif & & \\
\hline
\end{tabular}

\section{NOTES}

1. Les consonnes de la racine sont marquées par la lettre $C$ dans cette étude.

2. On trouve dans les productions journalistiques en arabe standard la présence d'un groupe prépositionnel introduit par la préposition min, qui rappelle le complément d'agent du français introduit par par ou de l'anglais introduit par by. Nous adressons cette question pour l'arabe du Caire dans la section 4.2 de cette étude.

3. « Kausativ (machen $z u$, bringen $z u$, veranlassen $z u$ ). »

4. Cairokee (كايروكي) est un groupe pop-rock de la nouvelle scène musicale alternative cairote. Le groupe a gagné en notoriété en produisant des chansons contestataires lors de la révolution de janvier 2011.

5. La notion de ditransitivité renvoie au schéma syntaxique d'un verbe qui peut être construit avec deux objets directs. En termes sémantiques, ces deux objets directs peuvent renvoyer par exemple au thème et au destinataire de l'action décrite par le procès.

6. «Bei einer Reihe von transitiven Verben vom I. und II. Stamm kann die Objektsergänzung wegglassen werden, wodurch ein medio-passivischer Sinn eintritt. Das subject is nicht mehr Agens sonder Experiencer,

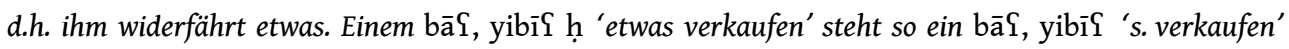
gegenüber: il Pusțuwāna bāSit kitīr 'die Plate hat sich gut verkauft' [SB]. Und so viele andere Verben, auch solche im II. Stamme. »

\section{RÉSUMÉS}

Cette étude s'intéresse aux interprétations reçues par les verbes de forme t-I en arabe du Caire, les interprétations passive et anticausative. Dans un premier temps, nous constatons d'une part que les verbes de forme t-I caractérisés par une transitivité inhérente ne reçoivent qu'une interprétation passive. D'autre part, la forme t-I n'existe pas lorsque la base de dérivation I est soit intransitive, soit ditransitive. L'interprétation passive est alors prise en charge respectivement par un verbe de forme $t$-II ou par une construction impersonnelle de la base de dérivation I. Lorsqu'un verbe de forme $t$-I peut recevoir les deux interprétations, passive et anticausative, trois critères sémantiques sont alors à prendre en compte pour appréhender cette alternance: le mode de réalisation du procès, la labilité verbale et la spontanéité du déclenchement de l'action. Enfin, la mise en rapport des verbes de forme $t-I$ avec la construction analytique du causatif permet de révéler les différentes interprétations qu'ils reçoivent, et les rapports qu'ils entretiennent avec les notions d'agent et de cause. 
This study deals with the interpretations received by verbs formed on the t-I pattern in Cairo Arabic, passive and anticausative interpretations. First, we note on the one hand that verbs of $t-I$ form characterized by an inherent transitivity receive only a passive interpretation. On the other hand, the $\mathrm{t}$-I form does not exist when the derivation base I is either intransitive or ditransitive. The passive interpretation is then supported respectively by a verb of form t-II or by an impersonal construction of the base of derivation I. When a verb of $\mathrm{t}$-I form can receive both interpretations, passive and anticausative, three semantic criteria are to be considered to apprehend this alternation: the mode of realization of the process, the verbal lability and spontaneity of action initiation. Finally, the linking of the verbs of $t-I$ form with the analytic construction of causative reveals the different interpretations they receive, and the relationships they maintain with the notions of agent and cause.

INDEX

Mots-clés : passif, anticausatif, morphosyntaxe, diathèse, arabe du Caire

Keywords : passive, anticausative, morphosyntax, diathesis, Cairo Arabic

\section{AUTEUR}

JULIE HASLÉ

CERMOM-INALCO 